\title{
CMEARTICLE
}

\section{Tinnitus - ringing in the ears}

Hui Bin Yvonne $\underline{\text { Chan }}^{1}$, BSc, David $\underline{\text { Low }}^{1,2}$, MBBS, MMed, Heng Wai Yuen ${ }^{1,2}$, MBBS, MMed, Choon How $\underline{\text { How }}^{1,3}$, MMed, FCFP

Robin, your patient for 30 years, visited your clinic with his wife Mary. You sensed that he was hesitant about sharing something. Upon further probing, he opened with "It's really nothing..." and went on to describe a soft buzzing sound in his ears at night that seemed to be worsening over the past few years. Mary added that he sometimes failed to respond to her when she spoke to him from another part of their flat. She also noticed that he had completely stopped listening to his favourite collections of classical music in the past year.

\section{WHAT IS TINNITUS?}

Tinnitus is a perception of ringing, buzzing or other noises in one or both ears in the absence of an external source. ${ }^{(1)}$ For the purposes of management, it is useful to first categorise tinnitus into pulsatile and non-pulsatile types. Non-pulsatile tinnitus is more common and can take on various qualities such as buzzing, ringing, crackling, humming or a cicada-like sound.

The pathogenesis of non-pulsatile tinnitus remains speculative. Current hypotheses revolve around the concept of neural aberrancy in the cochlea, auditory nerve or along central auditory pathways. As tinnitus is frequently accompanied by hearing loss, the clinical approach to such tinnitus is similar to that for hearing loss. Other notable associated symptoms include dizziness, aural fullness, aural discharge and otalgia.

Pulsatile tinnitus, which is less frequently encountered, can be classified into pulse synchronous and non-pulse synchronous types. The former arises largely from vascular aetiologies, while the latter is associated with middle ear or palatal muscle myoclonus.

\section{HOW RELEVANT IS THIS TO MY PRACTICE?}

A 2016 systematic review found that the prevalence of tinnitus in the selected studies, using a common definition, ranged from $11.9 \%$ to $30.3 \%$ globally. ${ }^{(2)}$ Large population studies found increased prevalence with increasing age, peaking between 60 and 69 years of age..$^{(3,4)}$ Males appear to be more affected than females. ${ }^{(3)}$ Local data from Singapore is scant. A study on noiseexposed workers showed a prevalence of $23.3 \%{ }^{(4)}$ It was also reported that the prevalence of hearing loss in Singapore military conscripts prior to enlistment was found to be 36.7 per $1,000 .{ }^{(5)}$

Effects of tinnitus on an individual's well-being include insomnia, cognitive effects, anxiety and psychological distress. ${ }^{(6)}$ A local study in 2010 showed that tinnitus patients who hear multiple sounds tend to have a poorer quality of life (QOL), as measured by the Tinnitus Handicap Inventory. ${ }^{(7)}$ The reasons for the drop in QOL cited in this series were a lack of control over tinnitus, frustration and stress. Interestingly, middle-aged patients aged $40-50$ years are most bothered by their tinnitus. ${ }^{(2)}$ It is believed that although tinnitus is more common in older patients, having competing health issues reduces the relative burden of tinnitus on their QOL.

Tinnitus carries economic consequences as well. It can reduce productivity and, together with hearing loss, result in significant disability and loss of income..$^{(8-10)}$

\section{WHAT CAUSES TINNITUS?}

The differential diagnoses of tinnitus are illustrated in Fig. 1.

\section{Non-pulsatile tinnitus}

There are primary and secondary forms of non-pulsatile tinnitus. Primary tinnitus is idiopathic and may or may not be associated with sensorineural hearing loss (SNHL). Secondary tinnitus accompanies various external, middle and inner ear pathologies. Common differentials to consider include presbycusis, noiseinduced hearing loss, ototoxic medications, ear wax impaction, middle ear pathologies, sudden sensorineural hearing loss, Ménière's disease, vestibular schwannoma and other disorders.

\section{Presbycusis}

This is also known as age-related hearing loss and occurs in the elderly. It is believed to be caused by an accumulation of insults to the inner ear (e.g. noise, ototoxicity), on a background of genetic predisposition. Typically, the onset and progression of tinnitus and hearing loss are gradual, with high frequencies affected first.

\section{Noise-induced hearing loss}

Noise exposure is another major cause of SNHL and tinnitus. The tinnitus often precedes subjective hearing loss and serves as an early indicator of cochlear hair cell dysfunction. ${ }^{(11)}$ Heavy equipment, environmental exposure or portable music devices that are played loudly for long periods of time are common sources of loud noises. Tinnitus from brief exposure, such as attending a loud concert, usually resolves. However, tinnitus 


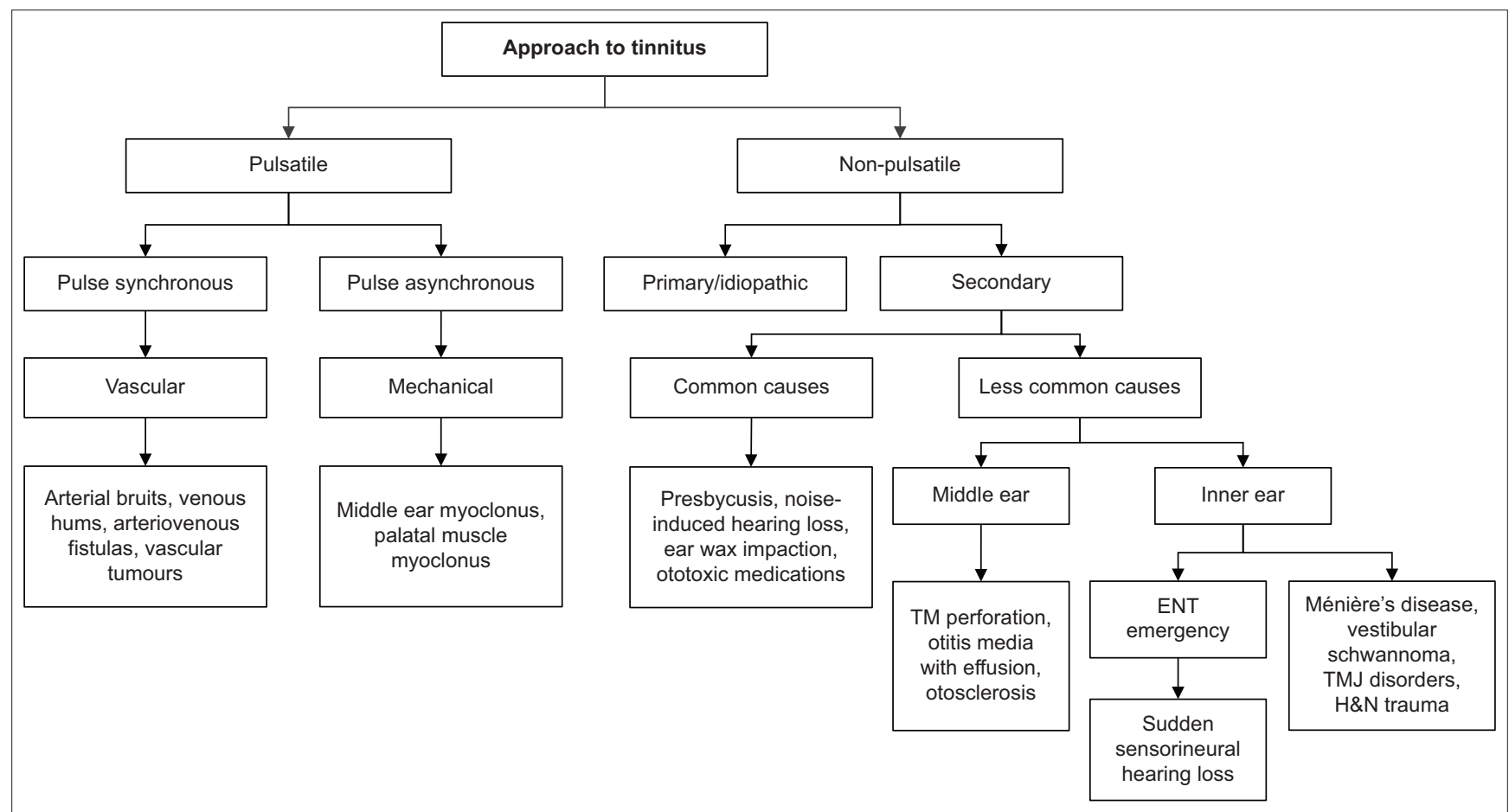

Fig. 1 Flowchart shows the approach to tinnitus. ENT: ear, nose and throat; H\&N: head \& neck; TM: tympanic membrane; TMJ: temporomandibular joint

from long-term or high-intensity exposure can be permanent. It is therefore important for physicians to actively seek these sources of exposure, as notification, workplace modification and appropriate protection can retard the progress of hearing loss.

\section{Ototoxic medications}

Several classes of drugs may cause or worsen tinnitus. Tinnitus may be the first symptom of ototoxicity and precede significant hearing loss and/or giddiness. The type of medication and dose prescribed determine the reversibility of ototoxicity and tinnitus. As such, eliciting a proper drug history and stopping the offending agent can save the patient from more severe ototoxicity. Such medications include antibiotics (e.g. aminoglycosides, vancomycin, macrolides), chemotherapeutic agents (e.g. cisplatin), diuretics (e.g. furosemide), aspirin and quinine.

\section{Ear wax impaction}

Patients with impacted ear wax that occludes the external auditory canal can present with tinnitus, conductive hearing loss and aural fullness. Symptoms usually resolve with clearance of the wax.

\section{Middle ear pathologies}

Middle ear pathologies such as tympanic membrane perforation, otitis media with effusion, and otosclerosis can also present with tinnitus. Most of these can be treated effectively with surgery. In the local context, adults should be screened for nasopharyngeal carcinoma when they present with otitis media with effusion.

\section{Sudden sensorineural hearing loss}

Sudden SNHL presents with a sudden drop in hearing that occurs within 72 hours. It is important to note that most patients complain of ear blockage rather than pure hearing loss, and thus it is important to think of this diagnosis when there is no obvious cause of ear blockage (e.g. ear wax, pus, debris). It is frequently associated with tinnitus in the affected ear. When there is accompanying vertigo, the syndrome is known as acute labyrinthitis. Most of these cases are idiopathic, with a small percentage due to vestibular schwannoma. These patients should be started on oral steroids early and referred to an otolaryngologist immediately to document their hearing loss and for possible specialised treatment such as intratympanic steroid injection.

\section{Ménière's disease}

Ménière's disease is a disorder of the inner ear characterised by recurrent episodic vestibular symptoms associated with SNHL, tinnitus and aural fullness. ${ }^{(12)}$ There is a consistent temporal relationship between these symptoms, and each episode lasts 20 minutes to hours.

\section{Vestibular schwannoma}

Vestibular schwannoma is a benign tumour of the vestibular nerves. It is confined to the internal auditory canal and/or cerebellopontine angle. Compression of the adjacent cochlear nerve results in unilateral tinnitus and asymmetrical hearing loss. Dizziness is not as pronounced, as these tumours are slowgrowing and allow for vestibular compensation. Older patients with vestibular schwannoma appear to suffer from more disabling tinnitus. ${ }^{(13)}$

\section{Other disorders}

Problems with the temporomandibular joint (TMJ) have been associated with tinnitus, but the exact mechanism is unknown. The tinnitus may improve when patients respond favourably to treatment for symptoms of TMJ dysfunction and craniocervical 
disease. ${ }^{(14)}$ Blunt head and neck trauma can also affect peripheral and central auditory pathways. Such injuries can cause tinnitus and non-specific dizziness.

\section{Pulsatile tinnitus}

\section{Pulse synchronous}

Pulse synchronous pulsatile tinnitus is often vascular in aetiology. Some common differentials to consider are arterial bruits, intracranial vascular malformations, vascular tumours and venous hums.

\section{$\underline{\text { Arterial bruits }}$}

Turbulent flow through atherosclerotic vessels in the neck, temporal bone and the cranium produces a bruit. Such patients complain of pulsatile tinnitus in one or both ears, but it is typically louder on the side of the pathology. They also usually have no other otologic complaints such as aural fullness or hearing loss, and their tinnitus is loudest in quiet environments.

\section{$\underline{\text { Intracranial vascular malformations }}$}

Congenital arteriovenous malformations are rarely associated with tinnitus, while the acquired-type arteriovenous fistula from head trauma or surgery is more symptomatic, ipsilateral to the fistula. Large dural arteriovenous fistula can cause devastating intracranial haemorrhages, and early detection is important.

\section{Vascular tumours}

Tympanojugular glomus tumours are the most common tumours of the middle ear. They arise from paraganglia cells over the jugular bulb or promontory. An important cause of pulsatile tinnitus, they present as a pulsating red mass in the middle ear on otoscopy. Although benign, they are locally aggressive. Management involves watchful waiting, surgery or radiotherapy.

\section{Venous hums}

Venous hums might be heard in patients with idiopathic intracranial hypertension or those with anomalies in their intracranial venous systems, including abnormally high placement of the jugular bulb or diverticulum of the sigmoid sinus. It is often described by patients as a soft, low-pitched hum that stops with a change of head position or compression at the base of the neck. ${ }^{(15,16)}$

\section{Non-pulse synchronous}

Non-pulse synchronous pulsatile tinnitus is commonly due to neuromuscular disorders such as middle ear myoclonus and palatal myoclonus. Myoclonus of the muscles of the middle ear (tensor tympani and stapedius muscles) or palate can be idiopathic or secondary to neuromuscular disorders such as multiple sclerosis. Such myoclonus is usually diagnosed from history, as patients are frequently asymptomatic at the time of consultation.

\section{WHAT CAN I DO IN MY PRACTICE?}

Physicians should determine the laterality, onset, temporal pattern and characteristics of the tinnitus by taking the history of the presenting complaint. Box 1 provides a brief guide to

\section{Box 1. A brief guide to history-taking:}

Associated symptoms

Ask about hearing loss, dizziness, aural fullness, aural discharge, otalgia and localising neurological symptoms.

\section{Past medical history}

Ask about cardiovascular risk factors and screen for mood disorders.

\section{Drug history}

Ask about exposure to ototoxic medications.

Family history

Ask for any history of brain tumours, or head and neck cancers.

\section{Social history}

Ask about occupational noise exposure.

Ideas, concerns, expectations

Distinguishing bothersome tinnitus from non-bothersome tinnitus can help to guide further management.

\section{Box 2. Tests to consider using during physical examination:}

- Otoscopy - look for pathology in the outer and middle ear

- Tuning fork tests - perform Weber's and Rinne's tests with a

$512-\mathrm{Hz}$ tuning fork to determine the type of hearing loss

- Neurological - focus on the gait, nystagmus, cranial nerves and cerebellar signs

- Neck - inspect and palpate lymph nodes and for masses such as tumours

For pulsatile tinnitus, examine the oral cavity for palatal myoclonus and auscultate over the chest, neck and skull for bruits.

history-taking. Box 2 lists the tests that can be used for physical examination.

\section{Management}

Initial management should be focused on resolving underlying causes. This includes ear syringing to clear ear wax, cessation of ototoxic medication and avoidance of exposure to loud noise. Patients should then be asked to maintain a healthy lifestyle with regular exercise and proper sleep, as cardiovascular health and stress levels can determine the severity of tinnitus. As most patients are most bothered by their tinnitus in quiet environments, they should be advised on the use of ambient noise from televisions, radios and tinnitus smartphone applications to 'mask' their tinnitus in such environments.

\section{WHEN SHOULD I REFER TO A SPECIALIST?} Referral to an otolaryngologist should be considered in the following scenarios:

- Any significant underlying cause is suspected from the clinical evaluation

- All cases of unilateral tinnitus, especially if accompanied by hearing loss, dizziness and other neurological deficits, while sudden hearing loss should be referred urgently to an otolaryngologist

- $\quad$ All pulsatile tinnitus

- Any persistent (> 6 months) or bothersome tinnitus ${ }^{(1)}$

An audiogram and a tympanogram are the first-line investigations in specialist practice. Other investigations such as radiological imaging and flexible nasopharyngoscopy are only 
ordered if deemed necessary. Magnetic resonance imaging of the internal acoustic canal is performed when patients present with unilateral symptoms, especially if accompanied by asymmetrical SNHL on the audiogram.

Similarly, management is first directed at any underlying cause. Further management then aims to reduce the impact of tinnitus on QOL. Bothersome tinnitus can be managed through a formal rehabilitation programme and with hearing aids. ${ }^{(1)}$ Tinnitus rehabilitation programmes involve a combination of education, counselling and sound therapy. This aims to reduce perception of the tinnitus and reduce its impact on the physical, social and mental well-being of patients. Hearing aids are an effective option for patients with both hearing loss and tinnitus. The perception of tinnitus is reduced, presumably through a reduction of neural aberrancy in peripheral and central auditory pathways.

The use of medications, such as antidepressants and anticonvulsants, and supplements (such as Ginkgo biloba, melatonin and zinc) has no proven efficacy and thus is not recommended in general.(1) However, antidepressants and anticonvulsants have roles in the management of concomitant mood disorders and specific types of tinnitus.

\section{TAKE HOME MESSAGES}

1. Tinnitus is a perception of sound in one or both ears in the absence of an external source, and has a prevalence of $11.9 \%-30.3 \%$ globally.

2. There are two types of tinnitus: pulsatile and non-pulsatile types, with the latter being more common.

3. Tinnitus can be accompanied by dizziness, aural fullness, aural discharge and otalgia.

4. Tinnitus may result in insomnia, cognitive effects, anxiety, psychological distress and lower QOL.

5. Common causes of non-pulsatile tinnitus include impacted ear wax, presbycusis, noise-induced hearing loss and ototoxicity.

6. Sudden SNHL presents with a sudden drop in hearing that occurs within 72 hours and frequently presents with tinnitus in the affected ear. This requires early medical assessment.

7. Initial management for tinnitus should focus on the identified underlying causes.

8. Most patients with tinnitus are bothered by it in quiet environments, and they can be advised to use ambient noise from televisions, radios and tinnitus smartphone applications to 'mask' their tinnitus.
9. A referral to an otolaryngologist is indicated if there are significant underlying causes suspected from the clinical evaluation, unilateral tinnitus, sudden hearing loss, pulsatile tinnitus, and any persistent or bothersome tinnitus.

You reviewed Robin three months later, after he had been fitted with a hearing aid. He thanked you for recommending him to get a hearing aid evaluation when he first visited you. It had helped him to enjoy his music once again, and he was also less disturbed by the buzzing sound now.

\section{REFERENCES}

1. Tunkel DE, Bauer $\mathrm{CA}$, Sun $\mathrm{GH}$, et al. Clinical practice guideline: tinnitus. Otolaryngol Neck Surg 2014; 151 (2 Suppl):S1-40.

2. McCormack A, Edmondson-Jones M, Somerset S, Hall D. A systematic review of the reporting of tinnitus prevalence and severity. Hear Res 2016; 337:70-9.

3. Møller AR. Epidemiology of Tinnitus in Adults. In: Møller AR, Langguth B, De Ridder D, Kleinjung T, eds. Textbook of Tinnitus. New York: Springer, 2011: 29-37.

4. Phoon WH, Lee HS, Chia SE. Tinnitus in noise-exposed workers. Occup Med 1993; 43:35-8.

5. Toh ST, Lu P, Ong M, Seet B. Prevalence of hearing disorders in Singapore military conscripts: a role for routine audiometry screening? Singapore Med J 2002; 43:622-7.

6. World Health Organisation. Burden of Disease from Environmental Noise: Quantification of Healthy Life Years Lost in Europe. Regional Office for Europe: World Health Organization, 2011.

7. Lim JJ, Lu PK, Koh DS, Eng SP. Impact of tinnitus as measured by the Tinnitus Handicap Inventory among tinnitus sufferers in Singapore. Singapore Med J 2010; 51:551-7.

8. Steinmetz LG, Zeigelboim BS, Lacerda AB, Morata TC, Marques JM. The characteristics of tinnitus in workers exposed to noise. Braz J Otorhinolaryngol 2009; 75:7-14.

9. Kim KS. Occupational hearing loss in Korea. J Korean Med Sci 2010; 25(Suppl):S62-9.

10. Henry JA, Dennis KC, Schechter MA. General review of tinnitus: prevalence, mechanisms, effects, and management. J Speech Lang Hear Res 2005; 48:1204-35

11. Le TN, Straatman LV, Lea J, Westerberg B. Current insights in noiseinduced hearing loss: a literature review of the underlying mechanism, pathophysiology, asymmetry, and management options. J Otolaryngol Head Neck Surg 2017; 46:41.

12. Goebel JA. 2015 Equilibrium Committee Amendment to the 1995 AAO-HNS Guidelines for the Definition of Ménière's Disease. Otolaryngol Head Neck Surg 2016; 154:403-4.

13. Baguley DM, Humphriss RL, Axon PR, Moffat DA. The clinical characteristics of tinnitus in patients with vestibular schwannoma. Skull Base 2006; 16:49-58.

14. Ren YF, Isberg A. Tinnitus in patients with temporomandibular joint internal derangement. Cranio 1995; 13:75-80.

15. Fortune DS, Haynes DS, Hall JW. Tinnitus. Current evaluation and management. Med Clin North Am 1999; 83:153-62.

16. Yeo WX, Xu SH, Tan TY, Low YM, Yuen HW. Surgical management of pulsatile tinnitus secondary to jugular bulb or sigmoid sinus diverticulum with review of literature. Am J Otolaryngol 2018; 39:247-52. 


\section{SINGAPORE MEDICAL COUNCIL CATEGORY 3B CME PROGRAMME} (Code SMJ 202009A)

1. Tinnitus is a perception of sound in one or both ears, in the absence of an external source.

2. Patients may complain that they hear not just ringing but also buzzing and clicking sounds.

3. The initial approach to tinnitus is to dichotomise tinnitus into pulsatile and non-pulsatile types.

4. Hearing loss is not a commonly associated symptom.

5. Pulse synchronous pulsatile tinnitus arises largely from mechanical causes, while non-pulse synchronous types are associated with vascular causes.

6. Tinnitus affects more females than males.

7. It is important to consider the effect of tinnitus on the patient's quality of life when managing the condition.

8. Common causes of tinnitus in our local context include impacted ear wax, presbycusis, noise-induced hearing loss and ototoxicity.

9. For presbycusis, hearing loss is gradual with low frequencies affected first.

10. It is important for physicians to actively seek a history of noise exposure, as workplace modification and appropriate protection can retard the progress of hearing loss.

11. Antibiotics such as macrolides can have the side effect of ototoxicity.

12. Sudden sensorineural hearing loss presents with a sudden drop in hearing that occurs within 48 hours.

13. Ménière's disease is characterised by recurrent episodic vertigo that is associated with hearing loss, tinnitus and aural fullness.

14. Patients who complain of pulse synchronous pulsatile tinnitus often find that it is louder on the contralateral side of the pathology.

15. Myoclonus of the muscles of the middle ear muscle or palate is always idiopathic.

16. Initial management should be focused on resolving underlying causes.

17. Patients can make use of ambient noise from televisions or radios to mask their tinnitus.

18. Sudden hearing loss need not be referred urgently.

19. Bothersome tinnitus can be managed through a formal rehabilitation programme and with hearing aids.

20. Hearing aids are not an effective option for patients with both hearing loss and tinnitus.

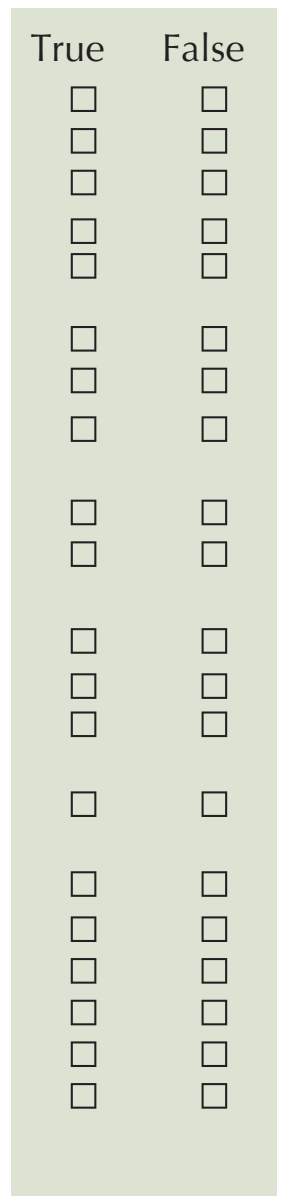

\section{Doctor's particulars:}

Name in full:

MCR no.:

Specialty:

Email:

\section{SUBMISSION INSTRUCTIONS:}

Visit the SMJ website: http://www.smj.org.sg/current-issue and select the appropriate quiz. You will be redirected to the SMA login page.

For SMA member: (1) Log in with your username and password (if you do not know your password, please click on 'Forgot your password?'). (2) Select your answers for each quiz and click 'Submit'.

For non-SMA member: (1) Create an SMJ CME account, or log in with your SMJ CME username and password (for returning users). (2) Make payment of SGD 21.40 (inclusive of $7 \%$ GST) via PayPal to access this month's quizzes. (3) Select your answers for each quiz and click 'Submit'.

RESULTS:

(1) Answers will be published online in the SMI November 2020 issue. (2) The MCR numbers of successful candidates will be posted online at the SMI website by 10 November 2020. (3) Passing mark is $60 \%$. No mark will be deducted for incorrect answers. (4) The SMJ editorial office will submit the list of successful candidates to the Singapore Medical Council. (5) One CME point is awarded for successful candidates. (6) SMC credits CME points according to the month of publication of the CME article (i.e. points awarded for a quiz published in the September 2020 issue will be credited for the month of September 2020, even if the deadline is in November 2020).

Deadline for submission (September 2020 SMJ 3B CME programme): 12 noon, 3 November 2020. 